\title{
Efecto del programa de rehabilitación cardiaca en la calidad de vida de pacientes con enfermedad coronaria
}

\author{
Effect of cardiac rehabilitation program on the quality of life of patients with coronary \\ heart disease \\ Óscar M. Hernández-Vásquez, Carlos J. Jaramillo-Gómez, Elsa M. Vásquez-Trespalacios, \\ María J. Orrego-Garay, Laura Duque-González y Mauricio Duque-Ramírez* \\ Centro de Medicina y Rehabilitación Cardiaca (CEMDE), CES Cardiología, Universidad CES, Medellín, Antioquia, Colombia
}

\section{Resumen}

Objetivo: Evaluar el efecto del programa de rehabilitación cardiaca en la calidad de vida, la clase funcional y la tolerancia al ejercicio de pacientes con enfermedad coronaria en un centro médico de la ciudad de Medellín. Método: Estudio descriptivo, longitudinal, comparativo antes y después, del cambio en la calidad de vida, la clase funcional y la tolerancia al ejercicio de pacientes con enfermedad coronaria sometidos a rehabilitación cardiaca. Resultados: Completaron 12 sesiones de rehabilitación 41 pacientes, de los que 28 eran hombres (68.3\%), con una edad promedio de $61.59 \pm 9.5$ años. La frecuencia cardiaca máxima lograda tuvo una reducción estadísticamente significativa $(p<0.0001)$. Tanto la clase funcional como la capacidad funcional no presentaron cambios significativos con el programa de rehabilitación; el peso y el índice de masa corporal tampoco se modificaron. Posterior al programa de rehabilitación se observó una mejoría significativa en los dominios de función física, dolor, salud general, vitalidad y salud mental. Conclusiones: Luego del programa de rehabilitación se observaron mejorías en la calidad de vida de los participantes, en los dominios físicos, de dolor, salud general, vitalidad y salud mental.

Palabras clave: Rehabilitación cardiaca. Calidad de vida. Enfermedad coronaria. Clase funcional.

\section{Abstract}

Objective: To assess the effect of a cardiac rehabilitation program on the quality of life, functional status and exercise tolerance of patients with coronary heart disease in a medical center in the city of Medellín. Method: A descriptive, longitudinal study comparing the change in quality of life, functional status and exercise tolerance of patients with coronary heart disease undergoing cardiac rehabilitation. Results: Twelve rehabilitation sessions were completed by 41 patients, 28 were men (68.3\%). The mean age was $61.59 \pm 9.5$ years. The maximum heart rate had a statistically significant reduction $(p<0.0001)$. Both functional status and functional capacity did not show a significant change with the rehabilitation program, weight and body mass index were neither modified. Significant improvement was observed in the domains of physical function, pain, general health, vitality and mental health after the rehabilitation program. Conclusions: The rehabilitation program improved quality of life, physical and pain domains, vitality and mental health of participants.

Key words: Cardiac rehabilitation. Quality of life. Coronary artery disease. Functional status.

\section{Correspondencia:}

*Mauricio Duque-Ramírez

E-mail: mauricioduquemd@gmail.com
Fecha de recepción: 27-08-2019

Fecha de aceptación: 16-04-2021

DOI: 10.24875/RCCAR.M21000051
Disponible en internet: 29-07-2021 Rev Colomb Cardiol. 2021;28(3):263-268 www.rccardiologia.com 0120-5633 / @ 2021 Sociedad Colombiana de Cardiología y Cirugía Cardiovascular. Publicado por Permanyer. Este es un artículo open access bajo la licencia CC BY-NC-ND (http://creativecommons.org/licenses/by-nc-nd/4.0/). 


\section{Introducción}

La enfermedad cardiovascular es la principal causa de mortalidad en el mundo. Cada año mueren aproximadamente 17.9 millones de personas debido a ella, lo que representa un $31 \%$ de todas las muertes, con el $75 \%$ de los casos en países de bajos y medianos recursos $^{1}$. Casi la mitad de las muertes por enfermedad cardiovascular están relacionadas con la cardiopatía isquémica. En Colombia, de acuerdo con las estadísticas publicadas por el Ministerio de Salud y Protección Social, la enfermedad isquémica del corazón es la principal causa de muerte tanto en hombres como en mujeres mayores de 45 años, e incluso supera a las muertes violentas y la mortalidad por cáncer ${ }^{2,3}$. Además, es una enfermedad con alto impacto económico. Solo en los Estados Unidos de América, para el año 2015 , los costos directos de salud atribuibles a enfermedad cardiovascular, sumados a los costos indirectos, se elevaron a 555 billones de dólares ${ }^{4}$. Por esta razón, todos los avances que se realicen en cuanto al tratamiento de dicha patología benefician tanto al paciente como a los sistemas de salud en general.

El manejo de la enfermedad coronaria requiere tratamiento farmacológico y no farmacológico. El no farmacológico incluye cambios en el estilo de vida y en la dieta, y un plan de ejercicio dirigido en programas de rehabilitación cardiaca. Dentro del proceso de recuperación de pacientes con síndrome coronario agudo, las recomendaciones incluyen la remisión y la asistencia regular a un programa de rehabilitación integral y dirigido. La American Heart Association recomienda que los programas de rehabilitación incluyan una evaluación basal del individuo, asesoramiento nutricional, estrategias para el control de factores de riesgo modificables (perfil lipídico, hipertensión, diabetes mellitus, obesidad y tabaquismo), consejo psicosocial, indicaciones de actividad física y entrenamiento físico ${ }^{5}$. Mediante este programa se espera mejorar la capacidad funcional de los pacientes, la calidad de vida y la tolerancia al ejercicio, e incluso disminuir el riesgo de muerte de causa cardiovascular ${ }^{6}$. Igualmente, se ha encontrado que por medio de programas de rehabilitación cardiaca se puede disminuir la carga económica que conllevan las enfermedades cardiovasculares?.

Se ha visto que la rehabilitación cardiaca después de un infarto es infrautilizada, especialmente por mujeres y ancianos. Además, un estudio encontró que solo se encuentra disponible en un $40 \%$ de los países con bajos-medianos recursos, donde la mayoría de los pacientes deben pagar por asistir a dicho programa ${ }^{8}$.
El objetivo de este estudio fue evaluar el efecto del programa de rehabilitación cardiaca en la clase funcional, la tolerancia al ejercicio y la calidad de vida de pacientes con enfermedad coronaria en un centro médico de la ciudad de Medellín, Colombia.

\section{Método}

\section{Diseño del estudio y participantes}

Se realizó un estudio descriptivo, longitudinal, con comparación antes y después de completar el programa de rehabilitación. La muestra del estudio estuvo constituida por personas mayores de 18 años que hubieran ingresado al programa de rehabilitación cardiaca de CEMDE por enfermedad coronaria. Se ingresaron los pacientes remitidos al programa de rehabilitación cardiaca de CEMDE entre los meses de abril y junio de 2016, como parte de su tratamiento por enfermedad coronaria.

Fueron incluidos en el estudio pacientes mayores de 18 años con diagnóstico de infarto de miocardio, angina, enfermedad coronaria evidenciada por coronariografía o sometidos a cirugía de revascularización coronaria. Todos debían aceptar voluntariamente y firmar su autorización para ingresar al estudio y al programa de rehabilitación.

Dentro del protocolo de la institución, al ingresar al programa los pacientes son sometidos a una prueba de esfuerzo que determina su capacidad funcional aeróbica al ingreso en MET (Metabolic Equivalent Time) y sirve para definir el plan de ejercicio que se seguirá. Se excluyeron del análisis los pacientes que no terminaron las 12 primeras sesiones de terapia de rehabilitación cardiaca.

\section{Instrumentos}

Todos los pacientes diligenciaron un formulario en el que se consignaron los datos generales (nombre, edad y sexo), la comorbilidad asociada (hipertensión arterial, diabetes mellitus, dislipidemia y tabaquismo) y el diagnóstico de ingreso al programa de rehabilitación (enfermedad coronaria, cirugía de revascularización miocárdica, revascularización percutánea con angioplastia o stent, o ambos).

Para la medición de la calidad de vida se utilizó el formulario SF36, y para la asignación del puntaje se utilizó el enfoque Rand que transforma el puntaje a una escala de 0 a 100. Así, en las ocho dimensiones del cuestionario, las preguntas de tres categorías se 
puntúan 0-50-100; las de cinco categorías se puntúan 0-25-50-75-100; y las de seis categorías se puntúan 0-20-40-60-80-100. Luego, los puntajes de ítems de una misma dimensión se promedian para crear los puntajes de las escalas que van de 0 a 100. Los ítems no respondidos no se incluyeron.

El cálculo de los MET logrados se efectuó de manera indirecta teniendo en cuenta la última etapa que alcanzó el paciente, la carga máxima (velocidad e inclinación en millas por hora) y el peso corporal de la persona, según el protocolo de Bruce. Con todos estos datos se calculó el consumo de oxígeno relativo alcanzado en mililitros por kilogramo por minuto, y luego se convirtió a MET (1 MET $=3,5 \mathrm{ml} / \mathrm{kg} / \mathrm{min})$. La clase funcional se determinó según los MET logrados en la primera y la última sesión de terapia de rehabilitación, medidos por el equipo de ergometría.

\section{Intervención}

El programa de rehabilitación cardiaca de CEMDE consta de 12 sesiones iniciales. Los pacientes ingresan al programa al ser remitidos de otras instituciones donde fueron atendidos por enfermedad coronaria. En la primera cita se les explica detalladamente en qué consiste el programa de rehabilitación, se realiza una entrega de material informativo sobre factores de riesgo y orientaciones básicas para el inicio del programa, y se hace énfasis en la necesidad de cumplir de manera adecuada el tratamiento farmacológico indicado al egreso de la hospitalización. En cada sesión, de acuerdo con la tolerancia, se aumenta progresivamente la cantidad de ejercicio, buscando incrementar la velocidad y el tiempo logrados en la banda con respecto a la sesión previa. Al finalizar la intervención, se miden de nuevo la calidad de vida, la clase funcional y la tolerancia al ejercicio de los pacientes.

\section{Análisis estadístico}

Se evaluaron las variables clínicas y sociodemográficas, la capacidad funcional, la tolerancia al ejercicio y la calidad de vida de los pacientes elegibles antes del inicio del estudio. Para evaluar el cambio en la calidad de vida y en los parámetros fisiológicos, antes y después de la rehabilitación, se utilizó la prueba de suma de rangos de Wilcoxon, y el cambio en las variables cualitativas se calculó con la prueba de $\mathrm{McNe}$ mar. El nivel de significancia estadística se estableció en $p<0.05$.

\section{Aspectos éticos}

El estudio fue aprobado por el Comité de ética de CEMDE. Siempre se promulgaron el respeto, la justicia y la beneficencia de los pacientes de acuerdo con las normas científicas, técnicas y administrativas para la investigación en salud dadas por el Ministerio de Salud en la Resolución \# 008430 del año 1993. De cada persona se obtuvo el consentimiento informado, después de explicarle los objetivos, los métodos, los posibles beneficios, los riesgos previsibles y la posibilidad de negarse a participar o retirarse en cualquier momento del programa de rehabilitación y del estudio.

\section{Resultados}

De todos los pacientes remitidos a terapia de rehabilitación, 16 no completaron las 12 sesiones y dos se negaron a participar en el estudio. Se presenta la experiencia de la aplicación de un programa de rehabilitación cardiaca en 41 pacientes con enfermedad coronaria remitidos al egreso de su hospitalización o desde la consulta externa al programa de rehabilitación de CEMDE. De estos 41 pacientes que completaron las primeras 12 sesiones establecidas, 28 eran hombres $(68.3 \%)$ y 13 mujeres (31.7\%). En la tabla 1 se muestran los datos de edad, peso e índice de masa corporal (IMC) de los pacientes.

Al completar las primeras 12 sesiones se evidenció una disminución del peso, de un promedio inicial de $71.9 \mathrm{~kg}$ a un promedio final de $71.68 \mathrm{~kg}$. Lo mismo sucedió con el IMC, que disminuyó de un promedio inicial de $26.79 \mathrm{~kg} / \mathrm{m}^{2}$ a un promedio final de $26.18 \mathrm{~kg} / \mathrm{m}^{2}$. $\mathrm{Si}$ bien tanto el peso como el IMC disminuyeron, la diferencia no fue estadísticamente significativa (Tabla 2). En los hombres, el peso inicial fue de 71.09 $\pm 8.98 \mathrm{~kg}$ y el peso final de $70.51 \pm 9.33 \mathrm{~kg}$, y el IMC inicial fue de $26.79 \pm 4.78 \mathrm{~kg} / \mathrm{m}^{2}$ y el final fue de 26.18 $\pm 6.08 \mathrm{~kg} / \mathrm{m}^{2}$. En las mujeres, el peso inicial fue de $73.70 \pm 12.95 \mathrm{~kg}$ y el final fue de $74.20 \pm 13.22 \mathrm{~kg}, \mathrm{y}$ el IMC inicial fue de $30.77 \pm 5.92 \mathrm{~kg} / \mathrm{m}^{2}$ y el final fue de $30.80 \pm 5.81 \mathrm{~kg} / \mathrm{m}^{2}$.

En cuanto a la capacidad funcional en MET, al completar las 12 sesiones no se encontró una variación estadísticamente significativa. Tampoco se modificó de forma estadísticamente significativa la clase funcional de la New York Heart Association (NYHA) ( $p=0.183$ ). La respuesta presora fue normal en todos los pacientes antes y después de completar las 12 sesiones de rehabilitación. La respuesta cronotrópica fue normal en 
Tabla 1. Características iniciales de los participantes

\begin{tabular}{|l|c|c|}
\hline Variable & Promedio & Rango \\
\hline Edad (años) & 61.59 & $42-83$ \\
\hline Peso (kg) & 71.9 & $49.7-102.2$ \\
\hline Índice de masa corporal $\left(\mathrm{kg} / \mathrm{m}^{2}\right)$ & 26.79 & $18.26-41.38$ \\
\hline
\end{tabular}

Tabla 2. Cambio en los parámetros fisiológicos

\begin{tabular}{|l|c|c|c|}
\hline Variable & $\begin{array}{c}\text { Promedio } \pm \\
\text { DE inicio }\end{array}$ & $\begin{array}{c}\text { Promedio } \pm \\
\text { DE final }\end{array}$ & p \\
\hline Peso promedio & $71.9 \pm 10.3$ & $71.68 \pm 10,6$ & 0.479 \\
\hline IMC promedio & $26.79 \pm 4.78$ & $26.18 \pm 6.0$ & 0.246 \\
\hline MET & $5.31 \pm 2.8$ & $5.52 \pm 2.3$ & 0.318 \\
\hline $\begin{array}{l}\text { Frecuencia cardiaca } \\
\text { máxima alcanzada }\end{array}$ & $110.12 \pm 24.2$ & $94.90 \pm 14.9$ & $<0.0001$ \\
\hline
\end{tabular}

DE: desviación estándar; IMC: índice de masa corporal; MET: Metabolic Equivalent Time.

Tabla 3. Cambios en las ocho dimensiones de calidad de vida

\begin{tabular}{|l|c|c|c|}
\hline Dimensión & $\begin{array}{c}\text { Promedio } \\
\text { DE inicio }\end{array}$ & $\begin{array}{c}\text { Promedio } \pm \\
\text { DE final }\end{array}$ & p \\
\hline Función física & $74.39 \pm 19.9$ & $76.58 \pm 18.7$ & 0.011 \\
\hline Rol físico & $39.02 \pm 40.3$ & $43.90 \pm 38.2$ & 0.155 \\
\hline Dolor corporal & $60.97 \pm 30.3$ & $68.71 \pm 23.9$ & 0.002 \\
\hline Salud general & $61.21 \pm 20.6$ & $65.24 \pm 16.4$ & 0.001 \\
\hline Vitalidad & $60.36 \pm 18.5$ & $62.80 \pm 17.5$ & 0.013 \\
\hline Función social & $64.02 \pm 28.2$ & $64.63 \pm 27.0$ & 0.577 \\
\hline Rol emocional & $69.10 \pm 44.9$ & $74.79 \pm 41.3$ & 0.102 \\
\hline Salud mental & $71.70 \pm 20.1$ & $77.26 \pm 17.5$ & $<0.0001$ \\
\hline
\end{tabular}

DE: desviación estándar.

el $100 \%$ de los pacientes antes y después de las primeras 12 sesiones de rehabilitación.

Al evaluar la frecuencia cardiaca, tras completar la rehabilitación se evidenció una disminución de la máxima alcanzada, en comparación con el valor máximo alcanzado en la primera sesión, con una variación estadísticamente significativa $(p<0.0001)$.

Al analizar las respuestas de calidad de vida se evidenció una mejoría en las ocho dimensiones del formulario (Tabla 3). De estas, fueron estadísticamente significativas la variación en función física, dolor corporal, salud general, vitalidad y salud mental. La dimensión de función física evalúa el grado en que la falta de salud limita las actividades físicas de la vida diaria, como el cuidado personal, caminar, subir escaleras, coger o transportar cargas, y realizar esfuerzos moderados e intensos. La dimensión de dolor corporal representa la medida de la intensidad del dolor padecido y su efecto en el trabajo habitual y en las actividades del hogar. La dimensión de salud general es la valoración personal del estado de salud, que incluye la situación actual, las perspectivas futuras y la resistencia a enfermar. La dimensión de vitalidad indica la energía frente al cansancio y el desánimo. La dimensión de salud mental general considera la depresión, la ansiedad, el autocontrol y el bienestar general. Para evitar un posible sesgo generado por el entrevistador, el cuestionario fue autoadministrado para de esta forma impedir las interpretaciones por parte del investigador.

\section{Discusión}

En el presente estudio se evidenció una reducción significativa de la frecuencia cardiaca máxima alcanzada con el ejercicio y una mejoría en la calidad de vida posterior a 12 sesiones de rehabilitación cardiaca basada en ejercicio.

Los estudios previos han demostrado una mejoría en la frecuencia cardiaca. En un estudio retrospectivo realizado en Austria ${ }^{9}$ se encontró, en un grupo de pacientes de sexo masculino, que la rehabilitación cardiaca se asoció con la normalización de la curva de rendimiento de la frecuencia cardiaca, que viene dada por la frecuencia cardiaca contra la intensidad de ejercicio en el tiempo. Teniendo en cuenta dicha curva en personas sanas y en pacientes que sobrevivieron a un infarto de miocardio, se puede interpretar que, alcanzado cierto nivel de intensidad de ejercicio, los enfermos tenían mayores cifras de frecuencia cardiaca en reposo que los sanos, y que posterior a un programa de ejercicio físico la frecuencia cardiaca de los pacientes disminuyó ${ }^{9}$. En el estudio de Szalewska, et al. ${ }^{10}$, en el cual se realizaron entre 19 y 22 sesiones de telerrehabilitación cardiaca en pacientes con enfermedad cardiovascular, se encontró una disminución no significativa en la frecuencia cardiaca al máximo esfuerzo, con respecto al inicio del estudio. En nuestro estudio, al evaluar la frecuencia cardiaca máxima alcanzada al completar la rehabilitación se evidenció una disminución respecto al valor inicial, con una 
variación estadísticamente significativa. Se plantea que esta respuesta es por la modulación de la regulación parasimpática.

En nuestro estudio no hubo diferencias estadísticamente significativas en cuanto a los MET logrados. Estudios previos, como en el de Golabchi, et al. ${ }^{11}$ realizado en Irán, sí encontraron mejoría en cuanto a los MET alcanzados luego de 24 sesiones de ejercicio, además que una disminución de la frecuencia cardiaca basal en estos pacientes. En el estudio retrospectivo de Gee et al. ${ }^{12}$, en los Estados Unidos de América, se encontró una mejoría significativa en los MET luego de 36 sesiones de rehabilitación cardiaca, especialmente en los hombres.

A diferencia de nuestros resultados, en un estudio realizado en pacientes con falla cardiaca crónica estable, en estadio $\mathrm{C}$ y clase funcional NYHA II-III, de diverso origen, solo se encontraron cambios en el estado de salud. Esta diferencia puede deberse a que no todos los participantes completaron todas las sesiones del programa de rehabilitación ${ }^{13}$.

No se observaron diferencias estadísticamente significativas en la mejoría de la clase funcional (NYHA). Sin embargo, en el estudio de Zhang et al. ${ }^{14}$ se halló una disminución estadísticamente significativa de la clase funcional NYHA tanto en los pacientes sometidos a rehabilitación cardiaca como en los que no tuvieron esta intervención. Esto puede deberse a que se realizaron más sesiones de ejercicio que en nuestro estudio.

En cuanto a la calidad de vida, también ha sido analizada en diversos estudios que han demostrado un beneficio posterior a la rehabilitación cardiaca. En el estudio de Gostoli, et al. ${ }^{15}$ se encontró beneficio en la percepción de la calidad de vida, expresado en la calidad de vida física, mental y total. En el estudio de Aldana et al. $^{16}$, en el que se empleó el cuestionario SF-36 en pacientes con enfermedad coronaria, se encontró una mejoría significativa en salud general, rol físico, función social, dolor corporal y salud mental, pero no en función emocional, en los pacientes que recibieron rehabilitación cardiaca; resultados similares a los de nuestro estudio. En nuestro estudio sí se evidenció mejoría en la dimensión de vitalidad. Finalmente, en una revisión sistemática Cochrane de 2016 sobre rehabilitación cardiaca en la enfermedad coronaria se encontró que en la mayoría de los estudios mejoró la calidad de vida relacionada con la salud $^{6}$.

La principal limitación de nuestro estudio fue la dificultad de los pacientes para poder asistir y cumplir las citas al programa de rehabilitación. Este en un factor que debe ser tenido en cuenta por las instituciones de salud para asegurar la asistencia a los programas y de esta forma mejorar las opciones de tratamiento en todos los pacientes. Otra limitación son las diferencias de las características basales de los pacientes. Finalmente, existen otros factores que pueden alterar los resultados, como la adherencia al tratamiento farmacológico, que podría influir en la frecuencia cardiaca de los pacientes.

En conclusión, la rehabilitación cardiaca es útil en el tratamiento de los pacientes con enfermedad coronaria en cuanto ayuda a mejorar parámetros de la calidad de vida y a modular la respuesta parasimpática medida como la frecuencia cardiaca ante un máximo esfuerzo.

\section{Financiamiento}

Los autores declaran que no existe financiamiento.

\section{Conflicto de intereses}

Se declara que no existe ningún conflicto de intereses.

\section{Responsabilidades éticas}

Protección de personas y animales. Los autores declaran que los procedimientos seguidos se conformaron a las normas éticas del comité de experimentación humana responsable y de acuerdo con la Asociación Médica Mundial y la Declaración de Helsinki.

Confidencialidad de los datos. Los autores declaran que han seguido los protocolos de su centro de trabajo sobre la publicación de datos de pacientes.

Derecho a la privacidad y consentimiento informado. Los autores han obtenido el consentimiento informado de los pacientes y/o sujetos referidos en el artículo. Este documento obra en poder del autor de correspondencia.

\section{Bibliografía}

1. Organización Mundial de la Salud. Enfermedades cardiovasculares. 2017. [Consultado el 30 de julio de 2019]. Disponible en: https:// www.who.int/es/news-room/fact-sheets/detail/cardiovascular-diseases-(cvds)

2. Ministerio de Salud y Protección Social. Indicadores básicos 2015. Situación de salud en Colombia. 2015. [Consultado el 31 de julio de 2019]. Disponible en: https://www.minsalud.gov.co/sites/rid/Lists/BibliotecaDigital/RIDE/VS/ED/GCFl/indicadores-basicos-en-salud-2015.pdf

3. Instituto Nacional de Salud. Enfermedad cardiovascular. Principal causa de muerte en Colombia. Boletín N.․ 1. Observatorio Nacional de Salud; 2013. Disponible en: https://www.ins.gov.co

\section{(1)}


Rev Colomb Cardiol. 2021;28(3)

4. Khavjou O, Phelps D, Leib A. Projections of cardiovascular disease prevalence and costs: 2015-2035. Technical report. RTI International 2016. [Consultado el 31 de julio de 2019]. Disponible en: https://healthmetrics.heart.org/wp-content/uploads/2017/10/Projections-of-Cardiovascular-Disease.pdf

5. O'Gara PT, Kushner FG, Ascheim DD, Casey DE, Chung MK de Lemos JA, et al. 2013 ACCF/AHA Guideline for the management of ST-elevation myocardial infarction: a report of the American College of Cardiology Foundation/American Heart Association Task Force on Practice Guidelines. J Am Coll Cardiol. 2013;61:e78-140.

6. Anderson L, Oldridge N, Thompson DR, Zwisler A-D, Rees K, Martin N et al. Exercise-based cardiac rehabilitation for coronary heart disease Cochrane systematic review and meta-analysis. J Am Coll Cardiol. 2016:67:1-12.

7. Hautala A, Kiviniemi A, Makikallio T, Koistinen P, Ryynanen O, Martikainen $\mathrm{J}$, et al. Economic evaluation of exercise-based cardiac rehabilitation in patients with a recent acute coronary syndrome. Scand J Med Sci Sports. 2016;27:1395-403.

8. Pesah E, Turk-Adawi K, Supervia M, López-Jiménez F, Britto R, Ding R, et al. Cardiac rehabilitation delivery in low/middle-income countries. Heart. 2019:105:1806-12.

9. Heber S, Sallaberger-Lehner M, Hausharter M, Volf I, Ocenasek H, Gabriel $\mathrm{H}$, et al. Exercise-based cardiac rehabilitation is associated with a normalization of the heart rate performance curve deflection. Scand $J$ Med Sci Sports. 2019;29:1364-74.
10. Szalewska D, Zieliński $P$, Tomaszewski J, Kusiak-Kaczmarek M Łepska L, Gierat-Haponiuk K, et al. Effects of outpatient followed by home-based telemonitored cardiac rehabilitation in patients with coronary artery disease. Kardiol Pol. 2015;73:1101-7.

11. Golabchi A, Basati F, Kargarfard M, Sadeghi M. Can cardiac rehabilitation programs improve functional capacity and left ventricular diastolic function in patients with mechanical reperfusion after ST elevation myocardial infarction? A double-blind clinical trial. ARYA Atheroscler. 2012;8:125-9.

12. Gee MA, Viera AJ, Miller PF, Tolleson-Rinehart S. Functional capacity in men and women following cardiac rehabilitation. J Cardiopulm Rehabil Prev. 2014;34:255-62.

13. Atehortúa DS, Gallo JA, Rico M, Durango L. Efecto de un programa de rehabilitación cardiaca basado en ejercicio sobre la capacidad física, la función cardiaca y la calidad de vida, en pacientes con falla cardiaca. Rev Colomb Cardiol. 2011;18:25-36.

14. Zhang $\mathrm{Y}$, Cao $\mathrm{H}$, Jiang $\mathrm{P}$, Tang $\mathrm{H}$. Cardiac rehabilitation in acute myocardial infarction patients after percutaneous coronary intervention: a community-based study. Medicine (Baltimore). 2018;97:e9785.

15. Gostoli S, Roncuzzi R, Urbinati S, Rafanelli C. Clinical and subclinical distress, quality of life, and psychological well-being after cardiac rehabilitation. Appl Psychol Health Well-Being. 2017;9:349-69.

16. Aldana SG, Whitmer WR, Greenlaw R, Avins AL, Thomas D, Salberg A, et al. Effect of intense lifestyle modification and cardiac rehabilitation on psychosocial cardiovascular disease risk factors and quality of life. Behav Modif. 2006;30:507-25. 